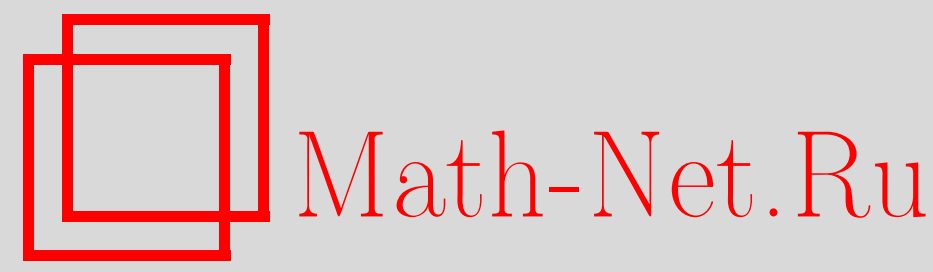

Ю. А. Бахтурин, М. В. Зайцев, С. К. Сегал, $G$-тождества неассоциативных алгебр, Матем. сб., 1999, том 190, номер 11, 3-14

DOI: https://doi.org/10.4213/sm437

Использование Общероссийского математического портала Math-Net.Ru подразумевает, что вы прочитали и согласны с пользовательским соглашением http://www . mathnet.ru/rus/agreement

Параметры загрузки:

IP: 54.197 .130 .99

26 апреля 2023 г., 11:01:05 


\title{
$G$-тождества неассоциативных алгебр
}

\begin{abstract}
Основньм классом алгебр, рассматриваемых в данной работе, являются алгебры лиевского типа. Он включает, в частности, ассоциативные алгебры, алгебры и супералгебры Ли, алгебры Лейбница, квантовые алгебры Ли и многие другие. Доказано, что если конечная группа $G$ действует на такой алгебре $A$ автоморфизмами и антиавтоморфизмами и $A$ при этом удовлетворяет существенному $G$-тождеству, то она удовлетворяет обычному тождеству, степень которого ограничена функцией от степени первоначального тождества и порядка $G$. Для случая обычных лиевских алгебр показано, что если $L$ - алгебра Ли, конечная группа $G$ действует на ней автоморфизмами и антиавтоморфизмами и порядок $G$ взаимно прост с характеристикой поля, то наличие тождества на кососимметричных элементах влечет наличие тождества на всей алгебре в целом с сохранением зависимости между степенями тождеств. Наконец, мы обобщаем теорему Амицура о полиномиальных тождествах в ассоциативных алгебрах с инволюцией на случай альтернативных алгебр с инволюцией.

Библиографиял: 9 названий.
\end{abstract}

\section{§1. Введение}

В нашей предыдушей статье [1] мы изучали так называемые $G$-тождества ассоциативных алгебр. Рассматривалась ситуация, когда на алгебре $A$ над полем $F$ конечная группа $G$ действует автоморфизмами и антиавтоморфизмами. Было показано, что если такая алгебра удовлетворяет существенному $G$-тождеству, то она удовлетворяет также обычному полиномиальному тождеству, степень которого ограничена некоторой функцией от степени $G$-тождества и порядка $G$. В качестве следствий мы получили усиление классических теорем Амицура [2], [3] о том, что любое нетривиальное $*$-тождество на ассоциативной алгебре с инволюцией $*$ влечет обычное тождество, и о том, что если подпространство $A_{+}$(соответственно $A_{-}$) симметричных (соответственно кососимметричных) элементов является PI-подпространством, то и сама алгебра $A$ является PI-алгеброй. В работах Амицура не было показано, что сушествует зависимость между степенями $*$-тождеств и обычных тождеств. Соответственно, техника Амицура не позволяла найти взаимосвязь между степенями тождеств на $A_{+}$или $A_{-}$и на самой алгебре $A$. В упомянутых работах авторов такая зависимость установлена, но более важно то, что техника доказательств является комбинаторной и может быть перенесена на другие классы алгебр.

Основным классом алгебр, рассматриваемых в данной работе, являются алгебры лиевского типа (см. [4]). Он включает, в частности, ассоциативные алгебры,

Первые два автора выполнили работу при частичной поддержке Российского фонда фундаментальных исследований (гранты № № 96-01-00146, 96-15-96050).

Третий автор выполнил работу при частичной поддержке NSERC (грант № A-5300).

(C) Ю.А. БАхтурин, М. В. ЗАЙцев, С. К. СегАЛ 1999 
алгебры и супералгебры Ли, алгебры Лейбница [5], квантовые алгебры Ли [6] и многие другие. Наш первый результат (теорема 2) утверждает, что если конечная группа $G$ действует на такой алгебре $A$ автоморфизмами и антиавтоморфизмами и $A$ при этом удовлетворяет сушественному $G$-тождеству, то она удовлетворяет обычному тождеству, степень которого ограничена функцией от степени первоначального тождества и порядка $G$.

Наш второй результат (теорема 3 ) утверждает, что если $L$ - алгебра Ли, конечная группа $G$ действует на ней автоморфизмами и антиавтоморфизмами и порядок $G$ взаимно прост с характеристикой поля, то наличие тождества на кососимметричных элементах влечет наличие тождества на всей алгебре в целом с сохранением зависимости между степенями тождеств.

Аналогичный результат неверен, если заменить кососимметричные элементы симметричньми.

ЗАмечАниЕ 1. Алгебра Ли с инволюцией может иметь нетривиальное тождество на подпространстве $L_{+}$и не иметь тождеств на всех своих элементах.

Дело в том, что любая алгебра Ли обладает инволюцией вида $x^{*}=-x$. В самом деле, $[x, y]^{*}=-[x, y]$ и $\left[y^{*}, x^{*}\right]=[-y,-x]=-[x, y]$. Но тогда все элементы алгебры кососимметричны и $x=0$ - тождество на симметричной части. Этот пример показывает также, что произвольное $*$-тождество на $L$ не всегда влечет обычное тождество.

Наконец, мы обобщаем теорему Амицура о полиномиальных тождествах в ассоциативных алгебрах с инволюцией (с ограничениями на степени) на случай альтернативных алгебр с инволюцией.

\section{§2. $G$-тождества}

Напомним основной результат работы [4]. Сначала введем необходимые понятия и обозначения. Пусть $Q$ - абелева группа. Алгебру $A$ над полем $F$ называют $Q$-градуированной, если $A$ является прямой суммой своих подпространств $A_{q}$, $q \in Q$, и $A_{q} A_{q^{\prime}} \subset A_{q+q^{\prime}}$. Элементы из $A_{q}$ называют однородными степени $q$. Мы говорим, что $Q$-градуировка конечна, если существует конечное подмножество $P$ в $Q$ такое, что $A_{q}=\{0\}$ для любого $q \notin P$. (Без потери обшности можно считать, что $0 \in P$.) Другими словами,

$$
A=\sum_{q \in P} A_{q}, \quad|P|=s<\infty .
$$

Далее, мы говорим, что $A=\sum A_{q}$ - алгебра лиевского типа, если для любых $g, h, k \in Q$ сушествуют такие скаляры $\alpha, \beta \in F$, что

$$
a(b c)=\alpha(a b) c+\beta(a c) b
$$

для всех $a \in A_{g}, b \in A_{h}, c \in A_{k}$, причем $\alpha \neq 0$. Если $\alpha=1, \beta=0$ для всех $g, h, k \in G$, то $A$-обычная ассоциативная алгебра с $Q$-градуировкой. Если $A-$ алгебра Ли над $F$, то $\alpha=1, \beta=-1$. Соотношения вида (1) выполняются в любой супералгебре Ли с $\mathbb{Z}_{2}$-градуировкой, а также в более общем классе цветных супералгебр Ли. Так назьваемые квантовые алгебры Ли (см. [6]) также удовлетворяют подобным соотношениям. Основной результат из [4] можно сформулировать следуюшим образом. 
TEOPEMA 1. Пyсmb

$$
A=\sum_{q \in Q} A_{q}
$$

- алгебра лиевского типа над произвольным полем $F$ с конечной $Q$-градуировкой, где $Q$ - некоторая группа. Предположим, что в нейтральной однородной компоненте $A_{0}$ выполняется нетривиальное тохсдество вида

$$
x_{0} x_{1} \cdots x_{d-1} \equiv \sum_{\sigma \in \operatorname{Sym}(d-1), \sigma \neq e} \alpha_{\sigma} x_{0} x_{\sigma(1)} \cdots x_{\sigma(d-1)},
$$

в котором $x_{0} x_{1} \cdots x_{d-1}$ и все $x_{0} x_{\sigma(1)} \cdots x_{\sigma(d-1)}$ - левонормированные коммутаторьи, а $\alpha_{\sigma} \in F$. Тогда сама алгебра А удовлетворяет нетривиальному тождеству вида (2).

Заметим, что в [4] этот результат был доказан в более обшем случае, когда $Q-$ произвольная полугруппа с единицей и с сокрашением.

Пусть теперь $A$ - алгебра лиевского типа и конечная группа $G$ действует на ней автоморфизмами и антиавтоморфизмами, причем любая однородная компонента $A_{q}$ переходит при действии элемента $g \in G$ в компоненту $A_{g \circ q}$, где о означает действие $G$ на $Q$ автоморфизмами и антиавтоморфизмами. Например, пусть $A=M_{n}(F)$ - алгебра матриц со стандартной целочисленной градуировкой:

$$
E_{i j} \in A^{k} \Longleftrightarrow j-i=k .
$$

Рассмотрим подгруппу $G$, порожденную в Aut* $A$ всеми сопряжениями $x \mapsto g x g^{-1}$, в которых $g=\operatorname{diag}\left(d_{1}, \ldots, d_{n}\right) \in G L(n, F)$, и транспонированием. Тогда, очевидно, $h\left(A_{k}\right) \subseteq A_{k}$, если $h$ - автоморфизм, и $h\left(A_{k}\right) \subseteq A_{-k}$, если $h$ - антиавтоморфизм. Другой естественный пример дает групповая алгебра $A=F G$ произвольной группы $G$. Нетрудно заметить, что $A-G$-градуированная алгебра и все внутренние автоморфизмы $G$ действуют автоморфизмами на алгебре $A$, переводя однородные в $G$-градуировке элементы в однородные.

Определим теперь $(G, Q)$-тождества на $A$.

Сначала разложим $G$ в объединение $G=G_{+} \cup G_{-}$, где $G_{+}$действует автоморфизмами, а $G_{-}-$антиавтоморфизмами. Пусть $H$ - векторное пространство с базисом из элементов $g \pi_{q}, g \in G, q \in Q$. Пусть $T=T(H)$ - тензорная алгебра $H$ (все тензоры с нулевым свободным членом). Пусть $X$ - некоторое множество и $\mathscr{F}=\mathscr{F}(X \mid G, Q)$ - линейная оболочка в свободной неассоциативной алгебре $T\langle X\rangle$ всех элементов вида $t_{v} v$, где $v$-мономы от элементов $X$, а $t_{v}$ - однородный тензор из $T$, причем $\operatorname{deg} v=\operatorname{deg} t_{v}$. Ясно, что $\mathscr{F}-$ подалгебра в $T\langle X\rangle$ и $\left(t_{u} u\right)\left(t_{v} v\right)=\left(t_{u} \otimes t_{v}\right)(u v)$.

Мы можем превратить $\mathscr{F}$ в $Q$-градуированную алгебру, положив $g \pi_{q} x \in \mathscr{F} g \circ q$ для любых $g \in G, q \in Q, x \in X$. Далее, по индукции, если $t_{u} u \in \mathscr{F}_{p}, t_{v} v \in \mathscr{F}_{q}$, то $\left(t_{u} \otimes t_{v}\right)(u v) \in \mathscr{F}_{p q}$. Тогда $\mathscr{F}_{p} \mathscr{F}_{q} \subset \mathscr{F}_{p q}$, как и необходимо.

Теперь зададим действие $G$ на $\mathscr{F}$, положив

$$
g *\left(\left(g_{1} \pi_{q_{1}} \otimes \cdots \otimes g_{s} \pi_{q_{s}}\right) u\right)=\left(\left(g g_{1}\right) \pi_{q_{1}} \otimes \cdots \otimes\left(g g_{s}\right) \pi_{q_{s}}\right) u,
$$

если $g \in G_{+}$, и

$$
g *\left(\left(g_{1} \pi_{q_{1}} \otimes \cdots \otimes g_{s} \pi_{q_{s}}\right) u\right)=\left(\left(g g_{s}\right) \pi_{q_{s}} \otimes \cdots \otimes\left(g g_{1}\right) \pi_{q_{1}}\right) u^{*}
$$


для $g \in G_{-}$, где $u^{*}$ - результат применения к $u$ стандартной инволюции на группоиде $\langle X\rangle$, продолжающей тождественное отображение на $X$ (т.е. $\left(x_{1}\left(x_{2} x_{3}\right)\right)^{*}=$ $\left.\left(\left(x_{3} x_{2}\right) x_{1}\right)\right)$. Из определения следует, что $g * \mathscr{F}_{q} \subset \mathscr{F}_{g \circ q}$. Например, в случае $(3)$ мы получаем

$$
g * \mathscr{F}_{\left(g_{1} \circ q_{1}\right) \ldots\left(g_{s} \circ q_{s}\right)} \subset \mathscr{F}_{g \circ\left(\left(g_{1} \circ q_{1}\right) \ldots\left(g_{s} \circ q_{s}\right)\right)}=\mathscr{F}_{\left(g g_{1} \circ q_{1}\right) \ldots\left(g g_{s} \circ q_{s}\right)}
$$

В этом случае мы пользовались тем, что $g$ действует на $Q$ как автоморфизм. Аналогично проверяется случай (4).

Рассмотрим теперь алгебру $A$ с $Q$-градуировкой, на которой группа $G=$ $G_{+} \cup G_{-}$действует так, что $g * A_{p} \subset A_{g \circ p}, G_{+}$действует автоморфизмами, а $G_{-}$- антиавтоморфизмами. Тогда любое отображение $\varphi: X \rightarrow A$ задает $Q$-градуированный $G$-гомоморфизм $\bar{\varphi}: \mathscr{F} \rightarrow A$, т.е. $\bar{\varphi}(\mathscr{F} p) \subset A_{p}, \bar{\varphi}(g * f)=g * \bar{\varphi}(f)$. Достаточно только положить

$$
\bar{\varphi}(f)=u\left(g_{1} * \pi_{q_{1}}\left(a_{1}\right), \ldots, g_{s} * \pi_{q_{s}}\left(a_{s}\right)\right)
$$

для $f=\left(g_{1} \pi_{q_{1}} \otimes \cdots \otimes g_{s} \pi_{q_{s}}\right) u\left(x_{1}, \ldots, x_{s}\right)$.

Здесь $u\left(x_{1}, \ldots, x_{s}\right)$ - это $x_{1} \cdots x_{s}$ с некоторой расстановкой скобок, а $\pi_{q}(a)$ обозначает $q$-проекцию $a \in A$ на $A_{q}$. Тогда как $f$, так и $\bar{\varphi}(f)$ являются однородными элементами степени $\left(g_{1} \circ q_{1}\right) \ldots\left(g_{s} \circ q_{s}\right)$. Далее, если $g \in G_{+}$, то

$$
\begin{aligned}
\bar{\varphi}(g * f) & =u\left(\left(g g_{1}\right) * \pi_{q_{1}}\left(a_{1}\right), \ldots,\left(g g_{s}\right) * \pi_{q_{s}}\left(a_{s}\right)\right) \\
& =g * u\left(g_{1} * \pi_{q_{1}}\left(a_{1}\right), \ldots, g_{s} * \pi_{q_{s}}\left(a_{s}\right)\right)=g * \bar{\varphi}(f) .
\end{aligned}
$$

Если $g \in G_{-}$, то

$$
\begin{aligned}
\bar{\varphi}(g * f) & =u^{*}\left(\left(g g_{s}\right) * \pi_{q_{s}}\left(a_{s}\right), \ldots,\left(g g_{1}\right) * \pi_{q_{1}}\left(a_{1}\right)\right) \\
& =g * u\left(g_{1} * \pi_{q_{1}}\left(a_{1}\right), \ldots, g_{s} * \pi_{q_{s}}\left(a_{s}\right)\right)=g * \bar{\varphi}(f),
\end{aligned}
$$

что и требовалось.

Рассмотрим теперь всевозможные отображения $\varphi: X \rightarrow A$ и все задаваемые ими в (5) $Q$-градуированные $G$-гомоморфизмы $\bar{\varphi}: X \rightarrow A$. Положим $I=\bigcap_{\varphi} \operatorname{Ker} \bar{\varphi}$. Любой элемент $f \in I$ назовем $Q$-градуированным $G$-тождеством $A$, или $(G, Q)$ тождеством $A$. Тогда верны все обычные результаты о тождествах: любое ненулевое тождество имеет ненулевое полилинейное (по $X$ ) следствие. Если поле скаляров бесконечно, то любое тождество эквивалентно системе однородных (по $X$ ) тождеств. В случае поля нулевой характеристики всякое тождество эквивалентно системе полилинейных по $X$ тождеств.

Возникает естественньй вопрос: верно ли, что нетривиальное $(G, Q)$-тождество влечет обычное тождество? В обшем случае ответ отрицателен даже для $G=\{e\}$ и $Q=\mathbb{Z}_{2}$. Например, тождество $\pi_{1} x=0$ выполняется в $A=A_{0} \oplus A_{1}$ с $A_{1}=\{0\}$, но в качестве $A$ может выступать любая алгебра. Рассмотрим другой пример. Пусть $Q$ - одноэлементная группа, $Q=\{0\}$ и произвольная группа $G$ действует на $A=A_{0}$ тождественными автоморфизмами. Тогда $g \pi_{0} x=\pi_{0} x-$ нетривиальное $(G, Q)$-тождество. Но и в этом случае $A$ может быть произвольной. Заметим также, что тождество нетривиально, так как оно не выполняется, например, в групповой алгебре групшы $G$. Если группа $Q$ бесконечна, например, $Q=\mathbb{Z}$ и число ненулевых слагаемых в $A=\sum_{q \in Q} A_{q}$ тоже бесконечно, то даже тождество $\pi_{0} x=0$ 
является слишком слабым условием. Например, абсолютно свободная алгебра $F\langle X\rangle$, градуированная степенями $X$, удовлетворяет этому тождеству. Наконец, пусть $G=\langle g\rangle_{\infty}$ - бесконечная циклическая группа и $\lambda \in F$ не является корнем из единицы. Тогда отображение $g: x \mapsto \lambda x \forall x \in X$ продолжается до автоморфизма алгебры $F\langle X\rangle$, который не имеет неподвижных точек. Этот пример показывает, что алгебра, на которой действует автоморфизмами бесконечная группа, может не иметь никаких нетривиальных тождеств, даже если подалгебра неподвижных точек удовлетворяет нетривиальному тождеству. Поэтому мы вынуждены ограничиться случаем, когда разложение $A=\sum_{q \in Q} A_{q}$ конечно и действуюшая группа $G$ также конечна.

Обозначим через $P \subset Q$ конечное подмножество $P=\left\{q_{1}, \ldots, q_{m}\right\}$ такое, что $A=\sum_{q \in P} A_{q}$. Обозначим через $\varepsilon$ элемент $e \pi_{q_{1}}+\cdots+e \pi_{q_{m}} \in H$. Тогда, продолжая отображение $x \mapsto a$, мы получим $\varepsilon x \mapsto a$. Положим $\varepsilon_{n}=\underbrace{\varepsilon \otimes \cdots \otimes \varepsilon}_{n}$. Тогда любое обычное тождество алгебры $A$ является и $(G, Q)$-тождеством. Оно имеет форму

$$
\sum_{v} \alpha_{v} \varepsilon_{n} v\left(x_{1}, \ldots, x_{n}\right)=0, \quad \alpha_{v} \in F
$$

где $v=v\left(x_{1}, \ldots, x_{n}\right)$ - одночлен из $F\langle X\rangle$. Если $A$ удовлетворяет соотношению (6), то для любых $a_{1}, \ldots, a_{n} \in A$ имеем

$$
\begin{aligned}
\sum_{v} \alpha_{v} v\left(e \pi_{q_{1}}\left(a_{1}\right)+\cdots+e \pi_{q_{m}}\left(a_{1}\right), \ldots, e \pi_{q_{1}}\left(a_{n}\right)\right. & \left.+\cdots+e \pi_{q_{m}}\left(a_{n}\right)\right) \\
& =\sum_{v} \alpha_{v} v\left(a_{1}, \ldots, a_{n}\right)=0 .
\end{aligned}
$$

Вернемся теперь к алгебрам лиевского типа. Если $A$ - такая алгебра, то из (1) следует, что любой элемент алгебры $\mathscr{F}$ по модулю $I$ (т.е. по модулю $(G, Q)$-тождеств алгебры $A$ ) может быть записан как линейная комбинация левонормированных одночленов (мы покажем это позже). Это дает нам возможность предполагать, что если $A$ имеет какое-то нетривиальное тождество, то она имеет также тождество $f\left(x_{1}, \ldots, x_{n}\right)=0$, в котором левая часть - это полином вида

$$
f\left(x_{1}, \ldots, x_{n}\right)=\sum_{\sigma \in S_{n}} t_{\sigma} x_{\sigma(1)} \cdots x_{\sigma(n)},
$$

где $t_{\sigma} \in T$ и все одночлены левонормированы.

Объединяя подходы работ [4], [1], мы получаем

ОПРЕДЕЛЕНИЕ 1. $(G, Q)$-тождество вида (7) назьвается существенным, если $t_{1}$ равно -1 . Другими словами, сушественное тождество имеет вид

$$
\varepsilon_{n} x_{0} x_{1} \cdots x_{n}=\sum_{\sigma \neq 1} t_{\sigma} x_{0} x_{\sigma(1)} \cdots x_{\sigma(n)} .
$$

Теперь мы можем сформулировать основной результат нашей статьи. 
ТЕОРема 2. Пусть $L$ - алгебра лиевского типа с конечной $Q$-градуировкой, на которой группа $G$ действует автоморфизмами и антиавтоморфизмами. Если L удовлетворяет существенному $(G, Q)$-тождеству степени $d$, то она удовлетворяет обичному тождеству степени $D$ (такой же форми, что $u(8)$, но со скалярными коэффичиентами $\left.t_{\sigma}=\alpha_{\sigma} \varepsilon_{D}, \alpha_{\sigma} \in F\right)$, причем $D$ зависит только от $d,|G|$ и числа $|P|$ ненулевых слагаемых в $Q$-градуировке $A$.

ДокАЗАтЕльство. Зададим естественный порядок на $X=\left\{x_{1}, x_{2}, \ldots\right\}$, положив $x_{i}<x_{j}$, если $i<j$, и продолжим его лексикографически до частичного порядка на всех неассоциативных одночленах на $X$. Сначала докажем два технических результата, которые помогут нам сравнивать элементы из $\mathscr{F}$ по модулю определенного ранее идеала $I$.

Лемма 1. Пусть $b$, у и $z$-одночлень на $X$ степеней $k, m$ и $n$ соответственно. Тогда для любого $t \in H^{\otimes^{k+m+n}}$ существуют $t_{1}, t_{2}, t_{1}^{\prime}, t_{2}^{\prime} \in H^{\otimes^{k+m+n}}$ такие, что

$$
\begin{aligned}
& t b y z \equiv t_{1} b(y z)+t_{2} b z y(\bmod I), \\
& t b(y z) \equiv t_{1}^{\prime} b y z+t_{2}^{\prime} b z y(\bmod I) .
\end{aligned}
$$

ДокАЗАТЕЛьство. Пусть сначала $t$ будет тензором ранга один. Тогда

$$
t=t_{b} \otimes t_{y} \otimes t_{z}, \quad t_{b} \in H^{\otimes^{k}}, \quad t_{y} \in H^{\otimes^{m}}, \quad t_{z} \in H^{\otimes^{n}}
$$

и все $t_{b}, t_{y}, t_{z}$ ранга 1. Рассмотрим одночлен

$$
t b(y z)=\left(t_{b} b\right)\left(\left(t_{y} y\right)\left(t_{z} z\right)\right)
$$

Заметим, что $t b y z$ и $t b(y z)$ однородны по $Q$-градуировке и лежат в одной компоненте $\mathscr{F}_{q}$. Если $q \notin P$, то $t b y z, t b(y z) \in I$. Если же $q \in P$, то применяем (1). Поскольку $t_{b}, t_{y}, t_{z}$ имеют ранг 1 , элементы $t_{b} b, t_{y} y$ и $t_{z} z$ однородны по $Q$-градуировке в $\mathscr{F}$. Используя (1), получаем

$$
\begin{aligned}
t b(y z) & \equiv \alpha\left(t_{b} b\right)\left(t_{y} y\right)\left(t_{z} z\right)+\beta\left(t_{b} b\right)\left(t_{z} z\right)\left(t_{y} y\right) \\
& =\alpha\left(t_{b} \otimes t_{y} \otimes t_{z}\right) b y z+\beta\left(t_{b} \otimes t_{z} \otimes t_{y}\right) b z y=\alpha t b y z+\beta t^{\prime} b z y
\end{aligned}
$$

с $0 \neq \alpha \in F$. Следовательно, можно взять $t_{1}=\alpha^{-1} t, t_{2}=-\beta \alpha^{-1} t^{\prime}, t_{1}^{\prime}=\alpha t$, $t_{2}^{\prime}=\beta t$, и для тензора ранга 1 лемма доказана. Поскольку тензоры ранга 1 образуют базис $H^{\otimes^{k+m+n}}$, доказательство леммы 1 закончено.

Лемма 2. Пусть а-левонормированный одночлен степени $m$ по $X, y_{1}, \ldots$, $y_{n} \in X$ и $y_{1}>y_{2}, \ldots, y_{n}$. Тогда для любого $t \in H^{\otimes^{m+n}}$ мохно найти $t^{\prime} \in H^{\otimes^{m+n}}$ mакой, что

$$
t a y_{1} \cdots y_{n}-t^{\prime} a\left(y_{1} \cdots y_{n}\right) \equiv \sum t_{w} w \quad(\bmod I)
$$

где $t_{w} \in T$, все $w$ - левонормированнье одночлены, причем $w<a y_{1} \cdots y_{n}$. 
ДоказАТЕЛьство. Применим соотношение (9) из леммы 1 к $b=a, y=y_{1}$, $z=y_{2}$ :

$$
\operatorname{tay}_{1} y_{2} y_{3} \cdots y_{n} \equiv t_{1} a\left(y_{1} y_{2}\right) y_{3} \cdots y_{n}+t_{2} a y_{2} y_{1} y_{3} \cdots y_{n} \quad(\bmod I)
$$

Второе слагаемое левонормировано и меньше, чем $a y_{1} \cdots y_{n}$. Применим (9) к первому слагаемому при $b=a, y=y_{1} y_{2}, z=y_{3}$ :

$$
t_{1} a\left(y_{1} y_{2}\right) y_{3} \cdots y_{n} \equiv t_{11} a\left(y_{1} y_{2} y_{3}\right) \cdots y_{n}+t_{12} a y_{3}\left(y_{1} y_{2}\right) \cdots y_{n} \quad(\bmod I) \text {. }
$$

Согласно (10) второе слагаемое является суммой левонормированных одночленов вида $t^{\prime} w$, где $w=a y_{3} \cdots$ менњше, чем $a y_{1} \cdots y_{n}$. Повторяя эту процедуру, мы получаем требуемое выражение для $\operatorname{tay}_{1} \cdots y_{n}$ по модулю $I$.

Теперь пусть $m$ - некоторое натуральное число. Полилинейный левонормированный $(G, Q)$-одночлен $t w, t \in T, w=y_{0} y_{1} y_{2} \cdots y_{n}, y_{0}=x_{0}, y_{i} \in\left\{x_{1}, \ldots, x_{n}\right\}$, $i=1, \ldots, n$, назовем $m$-неразложимым, если найдутся $m$ индексов $1 \leqslant i_{1}<$ $i_{2}<\cdots<i_{m} \leqslant n$ таких, что $y_{i_{1}}>y_{k}$ для всех $i_{m} \geqslant k>i_{1}, y_{i_{2}}>y_{k}$ для всех $i_{m} \geqslant k>i_{2}$ и т. д. Например, для $i_{m-1}$ должно выполняться условие $y_{i_{m-1}}>y_{k}$ для всех $i_{m} \geqslant k>i_{m-1}$. В противном случае мы говорим, что $t w m$-разложсим.

Сначала покажем, что любой полилинейный левонормированньй одночлен из $\mathscr{F}$ есть линейная комбинация $m$-неразложимых одночленов по модулю $I$, где $m=d-1$ и $d$-степень существенного $(G, Q)$-тождества в $L$.

Проведем доказательство от противного. Пусть $t w$ - лексикографически минимальный контрпример, т.е. минимальньй $m$-разложимый одночлен, не выражающийся через $m$-неразложимые. Предположим, что $t w=t y_{1} \cdots y_{n}$ и индексы $i_{1}<\cdots<i_{m}$ задают $m$-разложение на $t w$. Тогда построим разбиение нашего слова на подслова, чтобы применить к нему сушественное тождество. Положим $a_{0}=y_{0} y_{1} \cdots y_{i_{1}-1}$, если $i_{1}>1$, и $a_{0}=y_{0}$ в противном случае. Далее, положим $a_{m+1}=R_{y_{i_{m}+1}} \cdots R_{y_{n}}$ при $i_{m}<n$ либо будем считать $a_{m+1}$ пустьм словом, если $i_{m}=n$, где $R_{j}$ означает правое умножение на $y_{j}$. Для всех $j=1, \ldots, m-1$ мы положим

$$
a_{j}=y_{i_{j}} y_{i_{j}+1} \cdots y_{i_{j+1}-1}
$$

и $a_{m}=y_{i_{m}}$. Применяя $m$ раз лемму 2 к $a_{0} a_{1}, a_{0} a_{1} a_{2}, \ldots, a_{0} a_{1} \cdots a_{m}$, мы получим, что

$$
t w \equiv \bar{t}\left(a_{0} a_{1} \cdots a_{m}\right) a_{m+1} \quad(\bmod I)
$$

для некоторого $\bar{t} \in H^{\otimes^{n+1}}$.

Алгебра $L$ удовлетворяет сушественному тождеству степени $d=m+1$ :

$$
\varepsilon_{m} x_{0} x_{1} \cdots x_{m}=\sum_{\sigma \neq 1} t_{\sigma} x_{0} x_{\sigma(1)} \cdots x_{\sigma(m)} .
$$

Элемент из правой части (11) может быть записан как сумма

$$
\left(t_{0} \otimes t_{1} \otimes \cdots \otimes t_{m} \otimes t_{m+1}\right) a_{0} a_{1} \cdots a_{m} a_{m+1}
$$

в которой $t_{0}, \ldots, t_{m}-$ тензоры ранга 1, т.е. $t_{i}$ имеет вид

$$
t_{i}=g_{1} \pi_{b_{1}} \otimes \cdots \otimes g_{k} \pi_{b_{k}} .
$$


В этом случае элемент $t_{i} a_{i}$ однороден и $t_{i} \in \mathscr{F}_{r_{i}}$, где $r_{i}=\left(g_{1} \circ b_{1}\right) \cdots\left(g_{k} \circ b_{k}\right)$. Если $r_{i} \notin P$, то $t_{i} a_{i} \in I$ и элемент (13) лежит в $I$. Предположим теперь, что все $r_{0}, r_{1}, \ldots, r_{m}$ лежат в $P$. Тогда отображение

$$
\varphi\left(g \pi_{r_{i}}\right)=\left(g \circ t_{i}\right) a_{i}, \quad \varphi\left(g \pi_{r}\right)=0, \quad r \neq r_{i}, \quad i=0, \ldots, m,
$$

может быть продолжено до $Q$-градуированного $G$-гомоморфизма $\bar{\varphi}$ такого, что $\bar{\varphi}\left(\varepsilon x_{i}\right)=t_{i} a_{i}$. Нетрудно заметить, что

$$
\bar{\varphi}\left(t_{\sigma} x_{0} x_{\sigma(1)} \cdots x_{\sigma(m)}\right)=t_{\sigma}^{\prime} a_{0} a_{\sigma(1)} \cdots a_{\sigma(m)} .
$$

Следовательно, из (12) вытекает, что (13) совпадает с суммой произведений вида $t_{\sigma}^{\prime} a_{0} a_{\sigma(1)} \cdots a_{\sigma(m)}$ по модулю $I$, в которых $\sigma \neq 1$. После подстановки в (11) мы получаем

$$
t w \equiv \sum_{1 \neq \sigma} \bar{t}_{\sigma} a_{0} a_{\sigma(1)} \cdots a_{\sigma(m)} a_{m+1} \quad(\bmod I) .
$$

Теперь рассмотрим одно из слагаемых $b=\bar{t}_{\sigma} a_{0} a_{\sigma(1)} \cdots a_{\sigma(m)} a_{m+1}$. По лемме 1 это сумма левонормированных одночленов от $y_{0}, y_{1}, \ldots, y_{n}$. Но так как $\sigma \neq 1$, а $i_{1}, \ldots, i_{m}$ задают $m$-разложение на $y_{1} \cdots y_{n}$, то любое слагаемое строго меншше, чем $t w=t y_{0} y_{1} \cdots y_{n}$. По выбору $t w$ (лексикографически минимальный контрпример) $b$ является линейной комбинацией $m$-неразложимых одночленов по модулю $I$. Следовательно, и сам $t w$ является подобной комбинацией, что противоречит его выбору.

Теперь рассмотрим все одночлены $\varepsilon_{n} x_{0} x_{1} \cdots x_{n}=\sum_{\sigma \neq 1} t_{\sigma} x_{0} x_{\sigma(1)} \cdots x_{\sigma(n)}$. По модулю $I$ они лежат в $F$-линейной оболочке

$$
W=\left\langle t x_{0} x_{\sigma(1)} \cdots x_{\sigma(n)}\right\rangle
$$

где $x_{0} x_{\sigma(1)} \cdots x_{\sigma(n)}-m$-неразложимое слово, $t=g_{0} \pi_{r_{0}} \otimes \cdots \otimes g_{n} \pi_{r_{n}}$, а $g_{0}, \ldots, g_{n}$ $\in G, r_{0}, \ldots, r_{n} \in P$. Следовательно, $\operatorname{dim} W \leqslant|G|^{n} \cdot|P|^{n} a_{m}(n)$, где $a_{m}(n)$ обозначает число $m$-неразложимых полилинейных слов от $n$ переменных. Известно (см. [1]), что существует такая функция $f(x, y)$ от двух переменных, что для любого натурального $m$ и любого $c>0$ выполнено неравенство $a_{m}(n)<n ! / c^{n}$, если $n>f(m, c)$. Следовательно, для $c=|G| \cdot|P|+1$ мы получаем $\operatorname{dim} W<n$ !, и элементы $\varepsilon x_{0} x_{\sigma(1)} \cdots x_{\sigma(n)}$ линейно зависимы по модулю $I$. Эта линейная зависимость и дает нам требуемое тождество на $L$.

\section{§3. Алгебры Ли с тождествами на кососимметричных инвариантах}

Эту часть статьи мы начинаем с изучения обычных алгебр Ли. Пусть $G$ - конечная группа, действуюшая на алгебре Ли $L$ автоморфизмами и антиавтоморфизмами. Пусть ${ }^{G} L$ обозначает подпространство косых инвариантов этого действия, т.е. если $g \in G$ - автоморфизм, то $g(x)=x$, иначе $g(x)=-x$.

Теорема 3. Пусть $L$ - алгебра Ли над полем $F$ характеристики, не равной двум. Если ${ }^{G} L$ удовлетворяет нетривиальному тождественному соотношению степени $d$ u $(\operatorname{char} F,|G|)=1$, то L также удовлетворяет нетривиальному тождеству, степень которого зависит только от $d$ и $|G|$. 
ДокАЗАТЕльство. Разложим $G$ в объединение $G=G_{+} \cup G_{-}$, где $G_{+}-$нормальная подгруппа из элементов, действующих на $L$ как автоморфизмы, а $G_{-}-$ класс элементов, действующих как антиавтоморфизмы. Пусть $M=L^{G_{+}}-$подалгебра неподвижных точек действия $G_{+}$. Тогда ${ }^{G} L \subset M$. Если мы докажем, что $M$ удовлетворяет нетривиальному тождеству, то теорема будет доказана, как следует из результатов работы [7]. В частности, если $G=G_{+}$, то ${ }^{G} L=M$, и теорема доказана. В противном случае найдется $g_{0} \in G \backslash G_{+}$. Тогда $G_{-}=g_{0} G_{+}$. Поскольку нормальная подгруппа $G_{+}$из $G$ действует на $M$ тривиально, мы получаем действие $G / G_{+} \cong\langle *\rangle_{2}$, заданное соотношением $x^{*}=g_{0}(x)$. При этом $M=M_{-} \oplus M_{+}$, где $M_{-}=\left\{x: x^{*}=-x\right\}$ и $M_{+}=\left\{x: x^{*}=x\right\}$. Тогда для любых $x, y \in M_{-}$имеем:

$$
[x, y]^{*}=g_{0}([x, y])=\left[g_{0}(y), g_{0}(x)\right]=\left[y^{*}, x^{*}\right]=[-y,-x]=-[x, y],
$$

т.е. $\left[M_{-}, M_{-}\right] \subset M_{-}$. Аналогично $\left[M_{-}, M_{+}\right] \subset M_{+},\left[M_{+}, M_{+}\right] \subset M_{+}$. Следовательно, построенное выше разложение для $M$ является $\mathbb{Z}_{2}$-градуировкой. При этом $M_{-}={ }^{G} L, M_{+}=L^{G}$. Теперь из теоремы 1 следует, что $M$ удовлетворяет тождеству, степень которого зависит только от $d$, и доказательство теоремы закончено.

Мы уже отмечали во введении, что в последней теореме нельзя заменить косые инварианты ${ }^{G} L$ на обычные $L^{G}$.

Ряд следствий может быть получен и для других алгебр лиевского типа.

СлеДСТВИЕ 1. Пусть $A$ - ассоциативная алгебра, на которой конечная группа $G$ действует автоморфизмами и антиавтоморфизмами. Пусть также $(|G|, \operatorname{char} F)=1$. Если ${ }^{G} A$ или $A^{G}$ удовлетворяет нетривиальному полиномиальному тождеству степени $d$, то и сама А удовлетворяет нетривиальному полиномиальному тохдеству, степень которого ограничена функиией от $d$ и $|G|$.

Для доказательства следствия 1 нам потребуются следующие результаты.

ПРЕДЛОЖЕНИЕ 1. Пусть $L$ - лиевская подалгебра ассоциативной алгебры А над полем $F$. Предположим, что существует нетривиальный ассоциативный полином $f=f\left(x_{1}, \ldots, x_{n}\right)$, тождественно равный нулю на әлементах из $L$ в $A$. Тогда найдется лиевский полином вида

$$
\sum_{\sigma \in S_{n}} a_{\sigma}\left[x_{0}, x_{\sigma(1)}, \ldots, x_{\sigma(n)}\right]
$$

который также равен нулю на L тохдественно.

Мы используем обозначение $[x, y]=x y-y x$ для элементов $x, y$ из ассоциативной алгебры.

ДОКАЗАТЕЛЬСТВо ПРЕДЛОЖЕНИЯ 1. Пусть $F\langle X\rangle$ - свободная ассоциативная алгебра со счетным множеством порождающих $X=\left\{x_{1}, x_{2}, \ldots\right\}$. Как и прежде, обозначим через $P_{n}$ подпространство всех полилинейных многочленов в $F\langle X\rangle$, зависящих от $x_{1}, \ldots, x_{n}$.

Рассмотрим множество $I$ всех слабых тождеств пары $(A, L)$, т.е. все ассоциативные полиномы, тождественно равные нулю на элементах из $L$ в $A$. Ясно, что $I-$ 
идеал в $F\langle X\rangle$. Стандартные рассуждения показывают, что $I$ содержит некоторый полилинейный полином вида

$$
\sum_{\sigma \in S_{n}} \alpha_{\sigma} x_{\sigma(1)} \cdots x_{\sigma(m)}
$$

Другими словами, выполняется включение

$$
x_{1} x_{2} \cdots x_{m}-\sum_{1 \neq \sigma \in S_{n}} \alpha_{\sigma} x_{\sigma(1)} \cdots x_{\sigma(m)} \in I .
$$

Как и в доказательстве теоремы 2 , рассмотрим множество $V^{(m)}$ всех $m$-неразложимых одночленов от $X$.

Лемма 3. $P_{n} \equiv V^{(m)} \cap P_{n}(\bmod I)$.

ДокАЗАТЕЛЬСТво. Если $n<m$, то любой одночлен из $P_{n} m$-неразложим. Поэтому можно рассматривать только случай $n \geqslant m$. Теперь предположим, что наше утверждение ложно и существует лексикографически минимальный $m$-разложимый одночлен $B=y_{1} \cdots y_{n}$, где $y_{1}, \ldots, y_{n}$ - некоторые элементы из $X$, такой, что $B \notin V^{(m)}+I$. Зафиксируем $m$-разложение на $B$, т.е. набор индексов $1 \leqslant i_{1}<$ $i_{2}<\cdots<i_{m} \leqslant n$, для которых $y_{i_{k}}>y_{t}, t=i_{k}+1, \ldots, i_{m}$.

Как и в доказательстве теоремы 2 , обозначим через $t_{0}$ ассоциативное произведение $t_{0}=y_{1} \cdots y_{i_{1}-1}$, если $i_{1}>1$. При $i_{1}=1$ мы будем считать $t_{0}$ пустым словом. Если $i_{m}<n$, то обозначим $t_{m+1}=y_{i_{m}+1} \cdots y_{n}$, иначе считаем $t_{m+1}$ пустым словом. Для всех $k=1, \ldots, m-1$ положим

$$
t_{k}=\left[y_{i_{k}}, y_{i_{k}+1}, \ldots, y_{i_{k+1}-1}\right]
$$

и $t_{m}=y_{i_{m}}$. Теперь, вычисляя $t_{0} t_{1} \cdots t_{m+1}$ в $F\langle X\rangle$, мы видим, что старший член этого полинома (в смысле лексикограффического порядка) равен $B=y_{1} \cdots y_{n}$. Следовательно,

$$
B-t_{0} t_{1} \cdots t_{m+1} \in V^{(m)}+I
$$

так как $B$ - минимальный контрпример. Но согласно (14)

$$
t_{0} t_{1} \cdots t_{m+1} \equiv \sum_{1 \neq \sigma \in S_{m}} t_{0} t_{\sigma(1)} \cdots t_{\sigma(m)} t_{m+1} \quad(\bmod I) .
$$

Заметим, что все одночлены в записи $t_{0} t_{\sigma(1)} \cdots t_{\sigma(m)} t_{m+1}$ лексикографически меньше, чем $y_{1} \cdots y_{n}$, если $\sigma \neq 1$, и мы получаем выражение для $B$ в виде линейной комбинации строго меньших одночленов по модулю $I$. По выбору $B$ все эти одночлены лежат в $V^{(m)}+I$, следовательно, $B \in V^{(m)}+I$. Это противоречие завершает доказательство леммы 3.

Теперь, как и в доказательстве теоремы 2 , заметим что существует такая функция $f(x, y)$ от двух переменных, что условие $n>f(m, c)$ влечет ограничение $a_{m}(n)<n ! / c^{n}$ для любых $m, c$, например, для $c=2$ (см. [1]). Следовательно,

$$
\operatorname{dim} \frac{P_{n+1}}{P_{n+1} \cap I}<\frac{(n+1) !}{2^{n}}<n !
$$

для некоторого $n$. Поэтому множество из $n$ ! левонормированных лиевских одночленов $\left[x_{0}, x_{\sigma(1)}, \ldots, x_{\sigma(n)}\right]$ не может быть линейно независимым по модулю $I$, и доказательство предложения 1 закончено. 
ДокАЗАТЕЛЬСтво СЛЕДСТВИЯ 1 . Если подпространство $A^{G}$ удовлетворяет тождеству $f\left(x_{1}, \ldots, x_{n}\right)$, то подпространство ${ }^{G} A$ удовлетворяет соотношению $f\left(\left(x_{1} \circ y_{1}\right), \ldots,\left(x_{n} \circ y_{n}\right)\right)=0$, так как для любых $x, y \in{ }^{G} A$ выполнено включение $x \circ y=x y+y x \in A^{G}$. Поэтому достаточно рассмотреть только случай косых инвариантов. Обозначим через $L$ алгебру Ли, полученную из $A$ введением обычной коммутаторной скобки. $\mathrm{K} L$ применима теорема 3 , так как ${ }^{G} L={ }^{G} A$ по предложению 1 удовлетворяет нетривиальному тождеству.

Частным случаем следствия 1 является теорема Амицура, утверждающая, что если подпространство симметричных (или кососимметричных) элементов ассоциативной алгебры $A$ с инволюцией удовлетворяет нетривиальному тождеству, то сама $A$ является РІ-алгеброй. Но, кроме этого, следствие 1 дает связь между степенями тождеств на $A, A_{+}, A_{-}$.

Укажем еще одно обобщение теоремы Амицура. Это обобшение относится к более широкому классу альтернативных алгебр с инволюцией. Инволюция на альтернативной алгебре $A$ определяется как обычно - это линейньй оператор порядка $2 *: A \rightarrow A$ такой, что $(x y)^{*}=y^{*} x^{*}$. Симметричная и кососимметричная части также определяются стандартным образом:

$$
A_{+}=\left\{a \in A: a^{*}=a\right\}, \quad A_{-}=\left\{a \in A: a^{*}=-a\right\} .
$$

Напомним определение альтернативной алгебры.

ОПРЕДЕлЕниЕ 2. Алгебра называется альтернативной, если любая ее двупорожденная подалгебра ассоциативна.

Хорошо известно, что если char $F \neq 2$, то относительно операции $x \circ y=x y+y x$ алгебра $A$ является йордановой алгеброй, которую обозначают как $A^{(+)}$. Более того, эта йорданова алгебра специальна, т.е. изоморфна йордановой подалгебре некоторой ассоциативной алгебры с аналогично заданным умножением о. В качестве этой ассоциативной алгебры можно взять подалгебру в End $A$, порожденную правьми умножениями $R_{a}: x \mapsto x a$. Отображение $a \mapsto R_{a}$ не является гомоморфизмом $A$ в End $A$, но это гомоморфизм йордановых алгебр.

Следуя [8], будем говорить, что соотношение $f\left(x_{1}, \ldots, x_{n}\right) \equiv 0$ - тождество алгебры $A$, если $f$ - так называемый существенныи альтернативный полином, т.е. после опускания скобок он преврашается в нетривиальный ассоциативный полином. В этом случае мы говорим, что $A$ - альтернативная РІ-алгебра.

ТеОрема 4. Пусть $A$ - альтернативная алгебра с инволюиией над полем $F$ характеристики, не равной 2. Если $A_{+}$или $A_{-}$удовлетворяют нетривиальному тождеству, то $А$ является альтернативной PI-алгеброй.

ДокАзАтЕЛьство. Во-первых, заметим, что если $f=f\left(x_{1}, \ldots, x_{n}\right)$ - тождество на косых элементах в $A$, то

$$
f\left(x_{1} y_{1}-y_{1} x_{1}, \ldots, x_{n} y_{n}-y_{n} x_{n}\right) \equiv 0
$$

- тождество на симметричных переменных $x_{1}, \ldots, x_{n}, y_{1}, \ldots, y_{n}$, поскольку $a b-b a \in A_{-}$для любых $a, b \in A_{+}$. Поэтому достаточно доказать теорему только для симметричного случая.

Нам потребуется следующее предложение, которое фактически содержится в главе 5 из [8]. 
ПрЕДЛОЖЕНИЕ 2. Пусть $A$ - альтернативная алгебра над полем $F, \operatorname{char} F$ $\neq 2$ и $J=A^{(+)}$- построенная по ней йорданова алгебра. Предположим, что некоторый существенный йорданов полином тождественно равен нулю на J. Тогда $J$-специальная йорданова алгебра с нетривиальным йордановым тождеством.

ДоказАТЕльство. Если обозначить через $R_{a}$ правое умножение на $a$ в $A$, то по леммам 12 и 14 главы 5 из [8] правые умножения на элементы из $J$ удовлетворяют нетривиальному ассоциативному тождеству в End $A$. Тогда, как и в предложении 1 , сушествует йорданов полином $f=f\left(x_{1}, \ldots, x_{n}\right)$, который равен нулю на $x_{1}=R_{a_{1}}, \ldots, x_{n}=R_{a_{n}}$ при любых $a_{1}, \ldots, a_{n} \in J$. Так как отображение $a \mapsto R_{a}$ является вложением йордановой алгебры $A^{(+)}$в $(\operatorname{End} A)^{(+)}$, то $f \equiv 0-$ нетривиальное йорданово тождество в $J$.

Чтобы завершить доказательство теоремы, заметим, что $A^{(+)}=A_{+}^{(+)} \oplus A_{-}^{(+)}$ - это $\mathbb{Z}_{2}$-градуировка на специальной йордановой алгебре, причем нулевая компонента удовлетворяет нетривиальному йорданову тождеству. Тогда согласно [9] в $A^{(+)}$вьполнено некоторое йорданово тождество. Его и можно взять в качестве нетривиального соотношения на $A$.

ЗАмЕчАниЕ. В случае йордановых алгебр одно из следствий работы [9] состояло в том, что если конечная разрешимая группа $G,(\operatorname{char} F,|G|)=1$, действует автоморфизмами на специальной йордановой алгебре $J$ и множество $J^{G}$ неподвижных точек удовлетворяет нетривиальному тождеству, то и сама $J$ будет йордановой PI-алгеброй. Поэтому получаем такое следствие.

СлЕДСтвИЕ 2. Пусть конечная разрешимая группа $G,(\operatorname{char} F,|G|)=1$, действует на альтернативной алгебре $A$ над полем характеристики, не равной 2 , автоморфизмами и антиавтоморфизмами. Если подпространство инвариантов $A^{G}$ удовлетворяет нетривиальному тождеству степени $d$, то сама алгебра А имеет нетривиальное тождество, степень которого зависит только от $|G|$ и d.

\section{Список литературы}

1. Bahturin Y., Giambruno A., Zaicev M. G-identities on associative algebras // Proc. Amer. Math. Soc. 1999. V. 127. № 1. P. 63-69.

2. Amitsur S. A. Rings with involution // Israel J. Math. 1968. V. 6. P. 99-106.

3. Amitsur S. A. Identities in rings with involutions // Israel J. Math. 1969. V. 7. P. 63-68.

4. Bahturin Y., Zaicev M. Identities of graded algebras // J. Algebra. 1998. V. 205. № 1 . P. 1-12.

5. Loday J.-L. Une version non commutative des algebres de Lie: Les algebres de Leibniz // Enseign. Math. (2). 1993. V. 39. № 3-4. P. 269-293.

6. Liu K. Quantum central extensions // C. R. Math. Rep. Acad. Sci. Canada. 1991. V. 13. № 4. P. 135-140.

7. Linchenko V. Identities of Lie algebras with actions of Hopf algebras // Comm. Algebra. 1997. V. 25. № 10. P. 3179-3187.

8. Жевлаков К.А., Шестаков И. П., Ширшов А.И., Слинько А.М. Кольца, близкие к ассоциативным. М.: Наука, 1978.

9. Бахтурин Ю. А., Зайцев M. В. Тождества специальных йордановых алгебр с конечной градуировкой // Вестн. МГУ. Сер. 1. Матем., мех. 1998. Т. 53. № 2. С. 28-31.

Московский государственнњй университет им. М.В.Ломоносова; университет Альберты, Эдмонтон
Поступила в редакцию 17.03.1999 